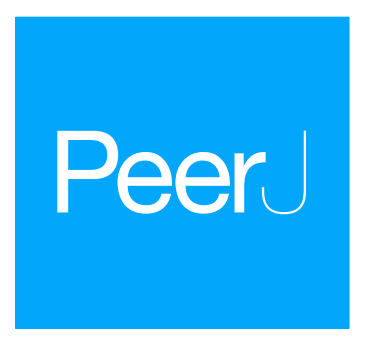

Submitted 2 July 2018

Accepted 16 October 2018

Published 19 December 2018

Corresponding author

Balázs Brankovics,

balazs.brankovics@wur.nl

Academic editor

David Ray

Additional Information and Declarations can be found on page 16

DOI 10.7717/peerj.5963

Copyright

2018 Brankovics et al.

Distributed under

Creative Commons CC-BY 4.0

\section{First steps towards mitochondrial pan- genomics: detailed analysis of Fusarium graminearum mitogenomes}

\author{
Balázs Brankovics ${ }^{1,2,3}$, Tomasz Kulik ${ }^{4}$, Jakub Sawicki ${ }^{4}$, Katarzyna Bilska ${ }^{4}$, \\ Hao Zhang ${ }^{5}$, G Sybren de Hoog ${ }^{2,3}$, Theo AJ van der Lee ${ }^{1}$, Cees Waalwijk ${ }^{1}$ and \\ Anne D. van Diepeningen ${ }^{1,2}$

\footnotetext{
${ }^{1}$ Wageningen Plant Research, Wageningen University \& Research, Wageningen, Netherlands

${ }^{2}$ Westerdijk Fungal Biodiversity Institute, Utrecht, Netherlands

${ }^{3}$ Institute for Biodiversity and Ecosystem Dynamics, University of Amsterdam, Amsterdam, Netherlands

${ }^{4}$ Department of Botany and Nature Protection, University of Warmia and Mazury, Olsztyn, Poland

${ }^{5}$ State Key Laboratory for Biology of Plant Diseases and Insect Pests, Institute of Plant Protection, Chinese Academy of Agriculture Sciences, Beijing, P.R. China
}

\section{ABSTRACT}

There is a gradual shift from representing a species' genome by a single reference genome sequence to a pan-genome representation. Pan-genomes are the abstract representations of the genomes of all the strains that are present in the population or species. In this study, we employed a pan-genomic approach to analyze the intraspecific mitochondrial genome diversity of Fusarium graminearum. We present an improved reference mitochondrial genome for F. graminearum with an intron-exon annotation that was verified using RNA-seq data. Each of the 24 studied isolates had a distinct mitochondrial sequence. Length variation in the F. graminearum mitogenome was found to be largely due to variation of intron regions (99.98\%). The "intronless" mitogenome length was found to be quite stable and could be informative when comparing species. The coding regions showed high conservation, while the variability of intergenic regions was highest. However, the most important variable parts are the intron regions, because they contain approximately half of the variable sites, make up more than half of the mitogenome, and show presence/absence variation. Furthermore, our analyses show that the mitogenome of $F$. graminearum is recombining, as was previously shown in F. oxysporum, indicating that mitogenome recombination is a common phenomenon in Fusarium. The majority of mitochondrial introns in F. graminearum belongs to group I introns, which are associated with homing endonuclease genes (HEGs). Mitochondrial introns containing HE genes may spread within populations through homing, where the endonuclease recognizes and cleaves the recognition site in the target gene. After cleavage of the "host" gene, it is replaced by the gene copy containing the intron with HEG. We propose to use introns unique to a population for tracking the spread of the given population, because introns can spread through vertical inheritance, recombination as well as via horizontal transfer. We demonstrate how pooled sequencing of strains can be used for mining mitogenome data. The usage of pooled sequencing offers a scalable solution for population analysis and for species level comparisons studies. This study may serve as a basis for future mitochondrial genome variability studies and representations. 
Subjects Bioinformatics, Genomics, Population Biology

Keywords Mitogenome, Comparative genomics, Mitogenomics, Pan-genome, Pool sequencing

\section{INTRODUCTION}

One of the most ideal markers for monitoring the distribution and spread of populations is the mitochondrial genome (Harrison, 1989; Taylor, 1986). Mitochondrial genomes are relatively small and, therefore, can be studied in their entirety. Due to its high copy number within individual cells, the mitochondrial genome is easy to access. Furthermore, it has a simple organization that makes homologous regions easy to identify. Finally, in many fungal groups mitogenomes are inherited uniparentally (Basse, 2010), which reduces the chance of heterologous recombination.

Mitochondrial sequences have been used for resolving phylogenetic and evolutionary relationships between fungi at all taxonomic levels (Liu et al., 2009; Avila-Adame et al., 2006; Fourie et al., 2013). In 2003, the DNA barcoding initiative started, aiming at using a single marker for taxon identification. The marker that was selected was a mitochondrial gene, cytochrome c oxidase I-COI or cox1 (Hebert et al., 2003). In Fusarium however, the use of $\operatorname{cox} 1$ was abandoned as a barcoding region, because the frequent presence of introns in the gene made this region impractical for PCR amplification (Gilmore et al., 2009). The difficulty in obtaining mitochondrial sequence, due to introns, lead to a general shift of interest from the mitochondrial to the nuclear genomes in fungi. Next generation sequencing (NGS) and new analysis methods have resolved this issue by dispensing with the need for PCR amplification for extracting mitochondiral sequences (Brankovics et al., 2016). In addition, de novo assembly of mitochondrial sequences from NGS data is not confounded by the presence of nuclear mitochondrial DNA segments (NUMTs), while NUMTs are known to cause problems in PCR-based barcoding (Song et al., 2008).

Fusarium graminearum is the major causative agent of Fusarium head blight (FHB), a devastating disease of small grain cereals. Besides reducing yield, the fungus contaminates crops with mycotoxins such as trichothecenes and zearalenone, which pose a serious threat to food and feed safety (Desjardins, 2006). Population studies of F. graminearum showed that the populations are highly dynamic and several displacements have been reported (Gale et al., 2007; Ward et al., 2008). Monitoring these population shifts is important, as they may differ in virulence, fungicide resistance and/or mycotoxin profile (Gale et al., 2007; Zhang et al., 2012).

The mitochondrial genome of $F$. graminearum encodes all genes typically associated with mtDNAs of filamentous fungi: two rRNA coding genes, 14 protein coding genes and a large set of tRNA coding genes (Al-Reedy et al., 2012). In addition, a large open reading frame with unknown function (LV-uORF) was found, flanked by tRNA genes. The first comparative studies of mitochondrial genomes of Fusarium spp. have revealed that F. graminearum has a significantly larger mitogenome than Fusarium spp. belonging to other species complexes analyzed so far (Fourie et al., 2013; Al-Reedy et al., 2012). Intron variation within the FGSC has not yet been analyzed, but the mitogenomes of different 
species within the F. fujikuroi species complex showed diversity in intron content based on the sequences of F. circinatum, F. fujikuroi and F. verticillioides (Fourie et al., 2013).

Most mitochondrial introns found in Fusarium are group I introns. These introns are selfsplicing ribozymes, which frequently contain homing endonuclease genes (HEGs) (Haugen, Simon \& Bhattacharya, 2005). The combination of intron and HEG forms a mobile element that is able to invade intronless copies of the "host" gene (Haugen, Simon \& Bhattacharya, 2005), thereby enabling horizontal spread of the mobile element through the population. This mechanism is called homing, since the homing endonuclease recognizes a target site of 15-45 bp, which makes the insertion highly sequence specific (Haugen, Simon \& Bhattacharya, 2005). A functional homing endonuclease is needed for the homing of the intron, but the intron may be retained as long as the self-splicing function of the intron is intact. Since the mitochondrial genes are crucial for the proper functioning of the cell, if an intron loses its ability to self-splice, then the intron is lost through precise excision (Goddard \& Burt, 1999). This mechanism allows an intron to spread in populations to strains that do not possess the given intron. This dispersion does not require further recombination. The mechanism does not allow one haplotype of an intron to replace another one, since the horizontal transfer is mediated only by the cleavage of an intronless copy. Hence, the replacement of one haplotype by another one can only be explained either by recombination or by loss of the original intron and insertion of the new haplotype.

Pan-genomes are the abstract representation of the genomes of all the strains that are present in the population. The idea of pan-genome or supra-genome comes from bacterial genomics, and originated from the distributed genome hypothesis (DGH) (Ehrlich, 2001; Tettelin et al., 2005). According to the DGH, each strain within a population/species contains a subset of contingency genes from within the supra-genome (pan-genome), i.e., the supra-genome is distributed among many individual strains (Ehrlich, 2001; Ehrlich, Hu \& Post, 2004). Pan-genome based analysis can be used to identify conserved, variable and strain specific regions within a group of genomes. Pan-genomes can be also employed to contrast two populations or two species.

In order to create a pan-genome for the mitogenome of F. graminearum, we have to better understand the nature and dynamics of the diversity in the mitochondrial genome of this organism. To accomplish this, a reliable reference has to be established as a basis for all comparative analyses. To this end, we resequenced the reference strain of F. graminearum, $\mathrm{PH}-1$, assembled its mitochondrial genome, improved its annotation and validated the annotation using RNA-seq. Subsequently, this reference was used to study the SNP frequencies, intron distribution and sequence variability of the different regions of the mitogenome within the species, by analyzing a total of 24 strains, which were individually sequenced, representing a wide range of hosts and geographic origins. Finally, we evaluated the efficacy of using pooled sequencing in assessing the mitogenome sequence diversity within a sample. Pooled sequencing offers the possibility of analyzing populations directly from field samples. 


\section{MATERIALS \& METHODS}

\section{Strains}

Thirteen F. graminearum strains were sequenced individually on the Illumina MiSeq platform (Table 1). In addition, F. graminearum strain PH-1 (CBS 123657, NRRL 31084) was sequenced on the Illumina HiSeq platform both as a single strain and as part of a pooled set of five F. graminearum strains (Table 1). Besides the newly sequenced strains, the whole genome sequencing reads of ten $F$. graminearum isolates were downloaded from the SRA database of NCBI that were produced by other research groups (Laurent et al., 2017; Wang et al., 2017). The outgroup, F. gerlachii strain was sequenced for an earlier publication (Kulik et al., 2016). A detailed description of the fungal strains is given in Table 1.

\section{Sequencing \\ Illumina MiSeq}

Whole genome libraries were prepared using the Nextera XT kit (Illumina, San Diego, CA, USA) from gDNA extracted from mycelium. The constructed libraries were sequenced on the Illumina MiSeq platform with $250 \mathrm{bp}$ paired-end read, version 2 . The fungal genomes were sequenced in a multiplexed format (6-7 samples per run), where an oligonucleotide index barcode was embedded within adapter sequences that were ligated to DNA fragments (Smith et al., 2010). Next, the sequence reads were de-multiplexed and filtered for low quality base calls, trimming all bases from $5^{\prime}$ and $3^{\prime}$ read ends with Phred scores $<$ Q30.

\section{Illumina HiSeq}

For F. graminearum strain PH-1 (CBS 123657, NRRL 31084) a random sheared shotgun library was prepared using the NEXTflex ChIP-seq Library prep kit with adaptations for low input gDNA according to the manufacturer's protocol (Bioscientific). The library was loaded as (part of) one lane of an Illumina paired-end flowcell for cluster generation using a cBot. Sequencing was done on an Illumina HiSeq2000 instrument using 101, 7, 101 flow cycles for forward, index and reverse reads respectively. De-multiplexing of resulting data was carried out using the Casava 1.8 software. Sequencing reads have been uploaded to the European Nucleotide Archive (ENA) with the accession number PRJEB18592.

The same method was applied for the pooled sequencing with the adjustment that random sheared shotgun library was prepared by using equal amounts of genomic DNA extract from all five strains (Table 1). Sequencing reads have been uploaded to the European Nucleotide Archive (ENA) with the accession number PRJEB18596.

\section{Third party sequencing data}

Besides the sequencing data that we have generated, we also made use of sequencing data produced by other research groups that had been submitted to SRA (Sequencing Read Archive) databases. This included a dataset of SRA data of six strains isolated from France (PRJNA295638; Laurent et al., 2017), three strains from China (PRJNA296400; Wang et al., 2017) and one strain from Australia (PRJNA235346; Gardiner, Stiller \& Kazan, 2014). The mitochondrial genome sequences for the strains sequenced by third parties are available 


\begin{tabular}{|c|c|c|c|c|c|}
\hline Species & Strain & Origin & Host & $\begin{array}{l}\text { Year of } \\
\text { isolation }\end{array}$ & $\begin{array}{l}\text { Sequenced } \\
\text { individually } \\
\text { or in a pool }\end{array}$ \\
\hline F. graminearum & $\begin{array}{l}\text { CBS123657 } \\
(\mathrm{PH}-1) \\
\text { NRRL31084 }\end{array}$ & USA & maize & 1996 & both \\
\hline F. graminearum & CBS119173 & USA & wheat head & 2005 & individually \\
\hline F. graminearum & CBS139513 & Argentina & barley & 2011 & individually \\
\hline F. graminearum & CBS139514 & Argentina & barley & 2010 & individually \\
\hline F. graminearum & CBS119799 & South Africa & wheat kernel & 1987 & individually \\
\hline F. graminearum & CBS119800 & South Africa & maize & 1990 & individually \\
\hline F. graminearum & CBS110263 & Iran & maize & 1968 & individually \\
\hline F. graminearum & CBS123688 & Sweden & oats & unknown & individually \\
\hline F. graminearum & CBS128539 & Belgium & wheat kernel & 2007 & individually \\
\hline F. graminearum & CBS138561 & Poland & wheat kernel & 2010 & individually \\
\hline F. graminearum & CBS138562 & Poland & wheat kernel & 2010 & individually \\
\hline F. graminearum & CBS138563 & Poland & wheat kernel & 2003 & individually \\
\hline F. graminearum & CBS104.09 & unknown & unknown & 1909 & individually \\
\hline F. graminearum & CBS185.32 & unknown & maize & 1932 & individually \\
\hline F. graminearum & CS3005 & Australia & barley & 2001 & individually \\
\hline F. graminearum & HN9-1 & China & wheat & 2002 & individually \\
\hline F. graminearum & HN-Z6 & China & wheat & 2012 & individually \\
\hline F. graminearum & INRA-156 & France & wheat & 2001 & individually \\
\hline F. graminearum & INRA-159 & France & wheat & 2001 & individually \\
\hline F. graminearum & INRA-164 & France & wheat & 2002 & individually \\
\hline F. graminearum & INRA-171 & France & wheat & 2001 & individually \\
\hline F. graminearum & INRA-181 & France & wheat & 2002 & individually \\
\hline F. graminearum & INRA-195 & France & wheat & 2002 & individually \\
\hline F. graminearum & YL-1 & China & wheat & 2012 & individually \\
\hline F. graminearum & bfb0999_1 & China & barley & 2005 & pooled \\
\hline F. graminearum & $68 \mathrm{D} 2$ & Netherlands & wheat & 2001 & pooled \\
\hline F. graminearum & CHG013 & China & maize & 2005 & pooled \\
\hline F. graminearum & CHG157 & China & barley & 2005 & pooled \\
\hline F. gerlachii & CBS123666 & USA & wheat head & 2000 & individually \\
\hline
\end{tabular}

in the Third Party Annotation Section of the DDBJ/ENA/GenBank databases under the accession numbers TPA: BK010538 -BK010547

\section{Assembly}

GRAbB was used with SPAdes assembler to reconstruct the mitogenome of the strains. GRAbB (Brankovics et al., 2016) was chosen because it is a wrapper program for iterative de novo assembly based on a reference sequence. SPAdes 3.8.1 (Bankevich et al., 2012; Nurk et al., 2013) assembler was used, since it offers good insight for the user into the relationship between nodes in the assembly graph and the relationship between nodes, contigs and 
scaffolds. The mitochondrial genomes were assembled from NGS reads using GRAbB by specifying the mitogenome sequence of $\mathrm{PH}-1$ strain (HG970331) as query sequence.

For each individually sequenced strain it was possible to resolve the assembly to a single circular sequence. When the GRAbB run finished for the strains that were pooled for sequencing, the final assembly graph was visualized using Bandage (Wick et al., 2015) and the assembly was resolved to two circular sequence variants to capture all the variation within the dataset (Text S1). For the first circular sequence, referred to as "short", the shorter alternative contigs were included in the path at each position where continuity was ambiguous. For the other sequence, referred to as "long", the longer alternatives were included. In this way, all the different sequence regions were represented at least once in the two sequences.

\section{Sequence annotation}

The initial mitogenome annotations were done using MFannot (http://megasun.bch. umontreal.ca/cgi-bin/mfannot/mfannotInterface.pl) and were manually improved: annotation of tRNA genes was improved using tRNAscan-SE (Pavesi et al., 1994), annotation of protein-coding genes and the $\mathrm{rnl}$ gene was corrected by aligning intronless homologs to the genome. Intron encoded proteins were identified using NCBI's ORF Finder (http://www.ncbi.nlm.nih.gov/gorf/gorf.html) and annotated using InterPro (Mitchell et al., 2015) and CD-Search (Marchler-Bauer \& Bryant, 2004). The annotated mitochondrial genome sequences are available under the following GenBank accession numbers: BK010538-BK010547, KP966550-KP966561, KR011238 and MH412632.

\section{Read mapping and SNP discovery}

The mitogenome of F. graminearum strain $\mathrm{PH}-1$ and the two mitogenome sequences obtained from the assembly of the pooled dataset were used as reference sequences for the read mapping and SNP discovery. The read mapping was done using aln and sampe subcommands of the Burrows-Wheeler Alignment tool (BWA-0.7.12-r1034) (Li \& Durbin, 2009). SNP calling was done using SAMtools mpileup (1.3.1) with $-g$ and $-f$ flag and BCFtools call (1.3.1) with - $m v$ flag (Li et al., 2009).

\section{Coverage analysis}

Coverage of different regions was estimated by, first, mapping reads of the pooled dataset to the reference sequence using the sampe subcommand of the Burrows-Wheeler Alignment tool (BWA-0.7.12-r1034) (Li \& Durbin, 2009). Then, read coverage was calculated using the genomecov command of bedtools v2.26.0. The following single copy nuclear protein coding genes were used to represent single copy nuclear regions: $\gamma$-actin (act), $\beta$-tubulin II (tub2), calmodulin (cal), 60S ribosomal protein L10 ( $r p l 10 a)$, the second largest subunit of DNA-dependent RNA polymerase II ( $r p b 2)$, translation elongation factor $1 \alpha(t e f 1 a)$, translation elongation factor 3 (tef3) and topoisomerase I (top1). The reference sequences were extracted from the genome of PH-1 (four chromosomes: HG970332, HG970333,

HG970334, and HG970335). The nuclear mitochondrial DNA segment (NUMT) used for coverage comparison was identified during the assembly of the pooled data (see Text S1). 


\section{Intron validation}

The RNA-seq data for F. graminearum PH-1 was downloaded from NCBI's SRA database, accession number PRJNA239711 (Zhao et al., 2014). Read mapping was done by HISAT2 aligner (Kim, Langmead \& Salzberg, 2015) by specifying putative intron positions. The intron position were validated based on the splice site output file and by examining the mapping SAM file produced by the aligner.

\section{Linear model}

$\mathrm{R}$ was used for linear model analysis to test whether the intron variation is the main reason of mitochondrial genome length variation within the species. The linear model was the following:

$y=x+c$

where $y$ was the total length of the mitochondrial genome, $x$ was the length of the intron sequences and $c$ was the $y$-intercept (average intronless length of the mitochondrial genomes). The $R^{2}$ value obtained from linear model analysis specifies what percentage of the variation of the dependent value (mitogenome length) is explained by the variation in the independent value (intron length).

$R^{2}=1-\frac{S S_{\text {residual }}}{S S_{\text {total }}}$

Residual sums of squares $\left(S S_{\text {residual }}\right)$ and total sums of squares $\left(S S_{\text {total }}\right)$ were calculated using the deviance function of $\mathrm{R}$.

\section{Comparative sequence analysis}

The nucleotide sequences were aligned using MUSCLE (Edgar, 2004a; Edgar, 2004b). Sequence variability of given regions was calculated by aligning the sequences. Then the number of characters with multiple character states was calculated and divided by the total number of characters in the alignment. This step was done using fasta_variability from the fasta_tools package (https://github.com/b-brankovics/fasta_tools).

\section{Detecting the presence of recombination}

The intergenic regions were analyzed using the $\Phi_{w}$-test implemented in SplitsTree (Bruen, Philippe \& Bryant, 2006) to detect whether there is recombination in the mitochondrial genome.

\section{RESULTS}

\section{Mitochondrial genome of $F$. graminearum}

The mitochondrial genomes of all 24 strains sequenced individually were assembled into single circular contigs. The re-sequencing of the mitochondrial genome of $F$. graminearum strain PH-1 revealed two SNPs compared to the most recent published mitogenome assembly (HG970331.1) of the strain that was based on next generation sequencing reads

(King et al., 2015). The correction of these SNPs was supported by the fact that all the other strains contained the same two SNPs obtained in the new assembly of PH-1. The newly 


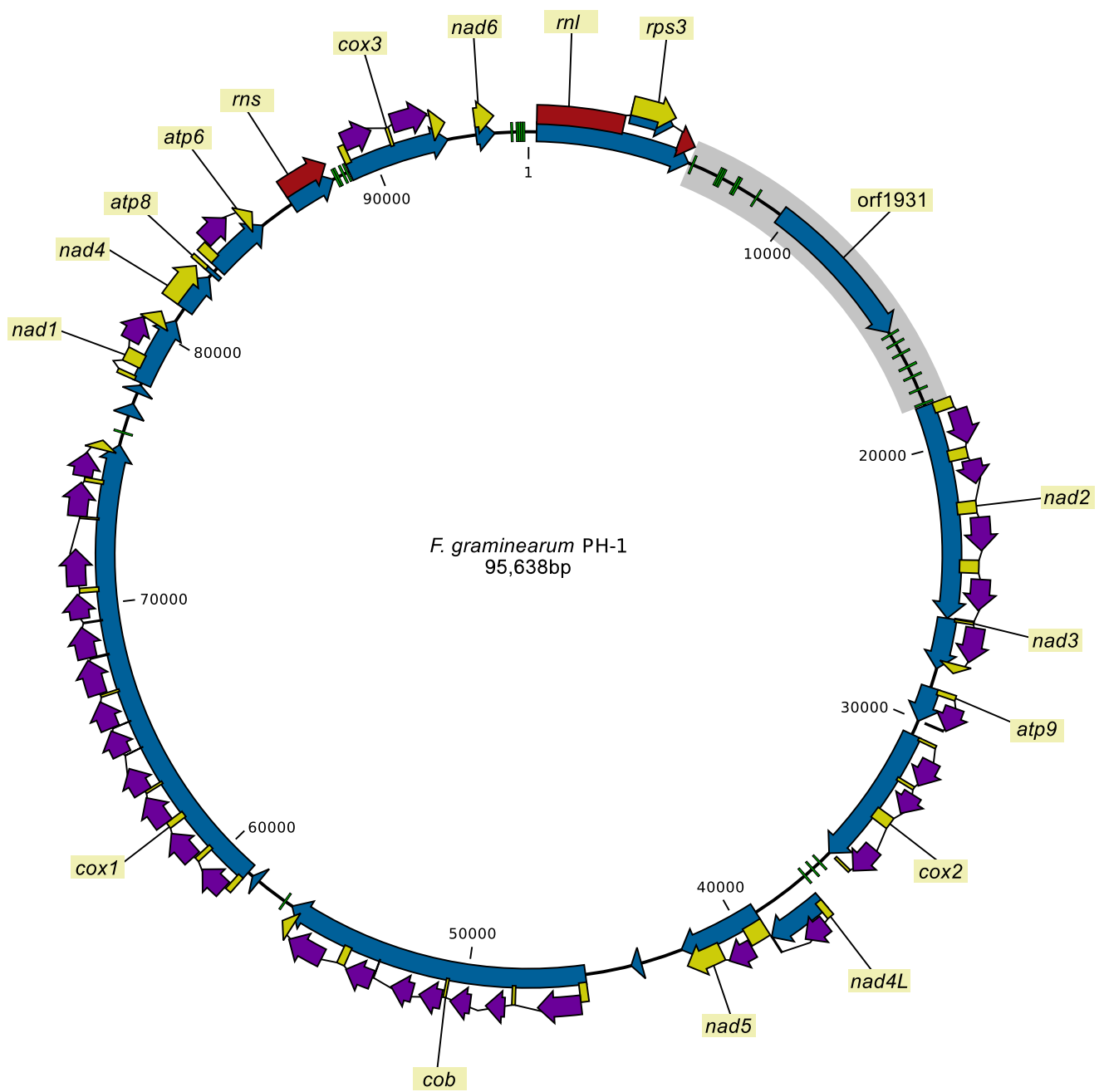

Figure 1 The mitogenome of $F$. graminearum strain PH-1. Green blocks: tRNA coding genes, blue arrows: genes or ORFs (no labels added for short ORFs), yellow arrows: protein coding sequences, red arrows: rDNA coding sequence, purple arrows: intron encoded homing endonuclease genes, gray box: the large variable (LV) region with orf1931 (LV-uORF).

Full-size DOI: 10.7717/peerj.5963/fig-1

assembled mitochondrial genome of the $\mathrm{PH}-1$ strain as well as the other mitochondrial genomes were annotated. The mitochondrial genomes of all strains contained the same set of genes in the same order and orientation (Fig. 1). To test whether the intron-exon models were predicted correctly, RNA-seq reads were mapped against the mitogenome of F. graminearum strain $\mathrm{PH}-1$. The results of the read mapping supported all of the predicted intron-exon boundaries.

\section{Mitogenome variability in $F$. graminearum}

The mitogenomes of F. graminearum strains analyzed showed variation in size, ranging from 93,560 bp to 101,424 bp (Table 2). To test whether intron variation is the main reason of mitochondrial genome length variation within the species, linear model analysis was 
Table 2 Mitochondrial genome variation of the Fusarium graminearum strains.

\begin{tabular}{|c|c|c|c|c|c|}
\hline Strain ID & $\begin{array}{l}\text { GenBank } \\
\text { accession } \\
\text { numbers }\end{array}$ & Size (bp) & Introns & $\begin{array}{l}\text { Intronic } \\
\text { (bp) }\end{array}$ & $\begin{array}{l}\text { Core } \\
\text { (bp) }\end{array}$ \\
\hline CBS123657 (PH-1) & MH412632 & 95,638 & 34 & 49,429 & 46,209 \\
\hline CBS185.32 & KP966550 & 96,300 & 34 & 50,120 & 46,180 \\
\hline CBS110263 & KP966551 & 97,364 & 35 & 51,165 & 46,199 \\
\hline CBS119173 & KP966552 & 100,342 & 37 & 54,130 & 46,212 \\
\hline CBS119799 & KР966553 & 96,005 & 35 & 49,919 & 46,086 \\
\hline CBS119800 & KP966554 & 97,462 & 35 & 51,280 & 46,182 \\
\hline CBS123688 & KP966555 & 95,035 & 34 & 48,837 & 46,198 \\
\hline CBS128539 & KP966556 & 96,134 & 35 & 49,996 & 46,138 \\
\hline CBS138561 & KР966557 & 95,034 & 34 & 48,837 & 46,197 \\
\hline CBS138562 & KP966558 & 99,062 & 36 & 52,865 & 46,197 \\
\hline CBS138563 & KP966559 & 99,068 & 36 & 52,865 & 46,203 \\
\hline CBS139514 & KР966560 & 96,167 & 35 & 49,980 & 46,187 \\
\hline CBS139513 & KP966561 & 95,041 & 34 & 48,837 & 46,204 \\
\hline CBS104.09 & KR011238 & 97,460 & 35 & 51,280 & 46,180 \\
\hline CS3005 & BK010538 & 93,560 & 33 & 47,381 & 46,179 \\
\hline HN9-1 & BK010539 & 96,307 & 35 & 51,567 & 44740 \\
\hline HN-Z6 & BK010540 & 97,767 & 34 & 50,120 & 47647 \\
\hline INRA-156 & BK010541 & 101,424 & 37 & 55,243 & 46181 \\
\hline INRA-159 & BK010542 & 96,199 & 35 & 49,996 & 46203 \\
\hline INRA-164 & BK010543 & 99,678 & 37 & 53,476 & 46202 \\
\hline INRA-171 & BK010544 & 96,199 & 35 & 49,996 & 46203 \\
\hline INRA-181 & BK010545 & 96,187 & 35 & 49,996 & 46191 \\
\hline INRA-195 & BK010546 & 97,358 & 35 & 51,165 & 46193 \\
\hline YL-1 & BK010547 & 97,996 & 36 & 51,777 & 46219 \\
\hline
\end{tabular}

Notes.

Core stands for the total mitogenome length minus the length of the intron regions.

used. The linear model that assumed that mitochondrial length variation is due only to variation of the length of intron regions explained $99.98 \%$ of intraspecific length variation observed in the data, showing that intron variation is the main reason behind intraspecific mitochondrial genome length variation. The standard deviation of the mitogenome length was $1,818 \mathrm{bp}$, which is $1.87 \%$ of the average mitochondrial genome length.

The coding regions (tRNA, rRNA and conserved protein coding genes) showed low levels of variation both within F. graminearum $(0.02 \%)$ and when compared to F. gerlachii $(0.02 \%)$. In addition, none of the SNPs found in protein coding regions caused amino acid substitution.

The large ORF with unknown function (LV-uORF) located in the large variable region of the mitogenome contained five SNPs within F. graminearum and the sequence in the F. gerlachii strain was identical to the most frequent haplotype within F. graminearum. All five SNPs resulted in amino acid substitution in the putative peptide sequences. The variability of the conserved protein coding regions was $0.02 \%$, while the variability of the 
Table 3 Distribution of variation in the intron and intergenic regions within and between species.

\begin{tabular}{llllllll} 
& \multicolumn{3}{c}{ Intraspecies } & & \multicolumn{3}{c}{ Interspecies } \\
\cline { 2 - 3 } & $\begin{array}{l}\text { Length } \\
(\mathbf{b p})\end{array}$ & $\begin{array}{l}\text { Variable } \\
\text { positions }\end{array}$ & $\begin{array}{l}\text { Variation } \\
\text { frequency }\end{array}$ & & $\begin{array}{l}\text { Length } \\
(\mathbf{b p})\end{array}$ & $\begin{array}{l}\text { Variable } \\
\text { positions }\end{array}$ & $\begin{array}{l}\text { Variation } \\
\text { frequency }\end{array}$ \\
\hline Coding & 21,572 & 4 & $0.02 \%$ & & 21,572 & 5 & $0.02 \%$ \\
Intron & 59,091 & 399 & $0.68 \%$ & & 59,091 & 419 & $0.71 \%$ \\
Intergenic & 18,982 & 310 & $1.63 \%$ & & 189,82 & 436 & $2.30 \%$ \\
\hline
\end{tabular}

LV-uORF region was $0.09 \%$ within F. graminearum. The difference in variability was even more striking on the protein sequence level, where the conserved protein genes showed no variation, while the $\mathrm{LV}-\mathrm{uORF}$ showed $0.26 \%$ variability.

The variability of the intergenic regions was $1.63 \%$ and $2.30 \%$ for intraspecies and interspecies, respectively. The overall sequence variability of intron sequences was $0.68 \%$ and $0.71 \%$ for intraspecies and interspecies, respectively. Although the variability of intron regions was significantly less than that of intergenic regions, both regions contained approximately equal numbers of variable sites (Table 3 ) due to the large length difference between the two regions. The intron regions were the most variable part of the mitochondrial genomes, because approximately half of the variable sites were located in introns, and introns were the only regions showing presence/absence variation within F. graminearum.

Interestingly, strains CBS 128539 and CBS 138561 had identical intergenic sequences, while strains CBS 104.09 and CBS 119800 (isolated 81 years apart) had identical intron sequences. However, each F. graminearum strain analyzed had a unique mitochondrial genome sequences.

\section{Intron patterns and recombination}

A total of 39 intron sites were found in the individually sequenced dataset (Table S1). Out of the 39 introns, 32 were present in all strains and 21 of these showed no variation at the intraspecies level and 14 at the interspecies level. The introns that showed presence/absence variation within the dataset were cob-i159, cob-i201, cox1-i1287, cox2-i228, cox2-i318, cox2-i552 and nad2-i1632 (Fig. 2 and Table S1). The intron names contain the gene name where they are located and the coding nucleotide position of the host gene after which they were inserted.

It was not possible to group the strains based on their intron patterns (presence/absence for each intron) without allowing for multiple gain or loss of introns (Table S1). This could be the result of recombination of parental mitochondrial genomes or the horizontal transfer of introns. Recombination would affect all regions equally, while the horizontal transfer of introns by homing would affect mostly the intron sequences and their immediate vicinity. Recombination of the intergenic regions was well supported $\left(p=2.26 * 10^{-6}\right)$ by the $\Phi_{w}$-test. 


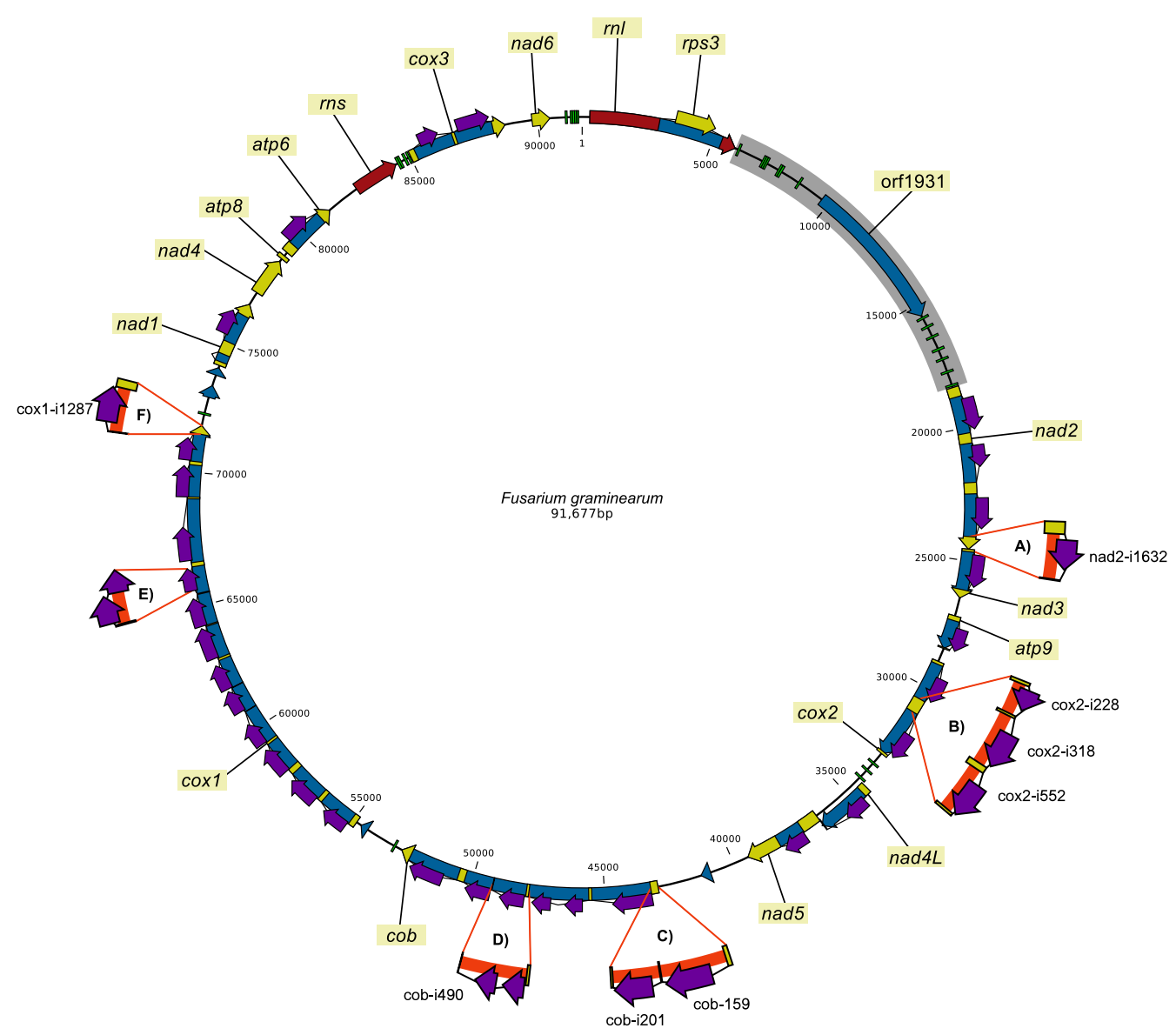

Figure 2 Pan-genomic representation of the presence/absence variation of introns in the mitochondrial genomes of the 24 F. graminearum strains. In the figure, the thick orange lines highlight intron sequences in the alternative sequences. (SNPs and short indels are not indicated.) (A) The insertion of nad2i1632; (B) the insertion of cox2-i228, cox2-i318 and cox2-i552; (C) the insertion of cob-i159 and cob-i201; (D) longer variant of cob-i490; (E) intron insertion in the HEG located in cox1-i906; and (F) the insertion of coxl-1287.

Full-size DOI: 10.7717/peerj.5963/fig-2

\section{Strategies to analyze pooled mitochondrial NGS data}

Two approaches were used to explore the mitogenome variability in the pooled dataset:

(i) assembling the reads de novo and (ii) mapping the reads to a reference sequence.

\section{De novo assembly approach}

The assembly resulted in a graph that contained five ambiguous sites that represented four insertion/deletion variations (three intron presence/absence variations for cob-i201, cox1-i1287, cox2-i318, and a large insertion inside the cob-i490 intron) in the dataset, and one site (located in nad4L-i239) where two different alleles were found in the strain set (Text S1). These polymorphic sites were too far apart to establish linkage between them, so two alternative assemblies were extracted from the assembly graph: one with the shorter allele at all of the positions and one with the longer allele at all of the positions (Text S1). The 
assembly method did not reveal SNP variations, only intron presence/absence variations and one replacement variation.

\section{Mapping approach}

To assess the influence of the reference sequence on the mapping and SNP calling results, three sequences were used as reference in independent mapping runs: both of the sequences obtained from the assembly approach of the pooled dataset were used as references, beside the curated mitogenome of the $\mathrm{PH}-1$ strain. Besides providing insight into the influence of the reference sequence to the downstream analysis, this also makes it possible to detect variation within intron sequences that may be absent in some of the reference sequences.

The lowest coverage detected for a single nucleotide allele was $21 \%$ of the reads that mapped to a given position. This is close to the expected value (20\%) for an allele present in a single strain in a pool of five strains. This result showed that the method was sensitive enough to detect a SNP present in a single strain. Furthermore, the results of all three analyses identified the same polymorphic sites. This means that the choice of reference sequence did not influence the SNP detection results.

The three runs of read mapping and SNP calling revealed a total of 15 SNPs (Table 4). The allele ratios were identical even when the reference sequence used for the mapping was different, with one exception: position 90,636. At this position both $\mathrm{PH}-1$ and the pooled assembly analysis showed $70 \%$ for the nucleotide present in the given reference and $30 \%$ for the alternative, despite the fact that the two references had different nucleotides at the given location (Table 4). Examination of the alignment of the reference sequences revealed that the sequence difference was not only a single nucleotide polymorphism at position 90,636 , but there was a 8 bp long indel at position 90,627-90,634. This nearby indel influenced the mapping of reads containing the allele differing from the reference sequence. This was the reason why the SNP calling skewed in favor of the reference allele in both mappings.

\section{Coverage analysis}

Coverage values were calculated for different genomic regions in order to determine whether coverage cutoffs could be used to differentiate between mitochondrial sequences and nuclear mitochondrial DNA segment (NUMT) sequences. The coverage of single copy nuclear regions that were present in all of the pooled strains was $290 \times$. The coverage of the NUMT sequence was $230 \times$, which suggests that it was present in four of the five pooled strains. The coverage of mitogenome regions that were present in all strains was $4,000 \times$. While, the coverage of singleton mitochondrial regions, present only in a single strain, was $475 \times$. The coverage gap was sufficiently large between shared single copy nuclear regions $(290 \times)$ and singleton mitochondrial sequence $(475 \times)$ to allow clear differentiation between them.

\section{DISCUSSION}

Comparative genomics analyses are traditionally reference (Laurent et al., 2017) or pairwise based (Fourie et al., 2013). Reference based methods are efficient at identifying regions that 
Table 4 List of single nucleotide polymorphisms identified in the pooled dataset of Fusarium graminearum strains. Positions are aligned positions between the $\mathrm{PH}-1$ reference sequence and the pooled sequences ("short" and "long"). "Reference" refers to the nucleotide found in the given reference sequence used for mapping, while "Alternative" refers to the nucleotide suggested by the mapped reads. Position 90,636 shows unusual ratios: in both mappings the reference nucleotide ( $\mathrm{C}$ or $\mathrm{A}$ ) has a frequency of $70 \%$ and the alternative nucleotide has $30 \%$. This is due to an adjacent indel that affects the mapping results.

\begin{tabular}{|c|c|c|c|c|}
\hline \multirow[b]{2}{*}{ Position } & \multicolumn{2}{|c|}{ PH-1 } & \multicolumn{2}{|c|}{ Pooled } \\
\hline & Reference & Alternative & Reference & Alternative \\
\hline 2,337 & $\mathrm{~A}(0.77)$ & $\mathrm{G}(0.23)$ & $\mathrm{A}(0.77)$ & $\mathrm{G}(0.23)$ \\
\hline 6,288 & $\mathrm{C}(0.41)$ & $\mathrm{A}(0.59)$ & $\mathrm{A}(0.61)$ & $\mathrm{C}(0.39)$ \\
\hline 6,355 & $\mathrm{~T}(0.42)$ & $\mathrm{C}(0.58)$ & $\mathrm{C}(0.60)$ & $\mathrm{T}(0.40)$ \\
\hline 13,540 & $C(0.78)$ & $\mathrm{A}(0.22)$ & $\mathrm{C}(0.78)$ & $\mathrm{A}(0.22)$ \\
\hline 37,126 & $\mathrm{C}(0.75)$ & $\mathrm{T}(0.25)$ & $\mathrm{C}(0.75)$ & $\mathrm{T}(0.25)$ \\
\hline 37,773 & $\mathrm{~A}(0.75)$ & $\mathrm{G}(0.25)$ & $\mathrm{A}(0.75)$ & $\mathrm{G}(0.25)$ \\
\hline 44,773 & $\mathrm{~A}(0.62)$ & $\mathrm{G}(0.38)$ & $\mathrm{A}(0.62)$ & $\mathrm{G}(0.38)$ \\
\hline 64,776 & $\mathrm{G}(0.53)$ & $\mathrm{A}(0.47)$ & $\mathrm{G}(0.53)$ & A $(0.47)$ \\
\hline 70,827 & $\mathrm{~A}(0.62)$ & $\mathrm{G}(0.38)$ & $\mathrm{A}(0.62)$ & $\mathrm{G}(0.38)$ \\
\hline 89,194 & $\mathrm{G}(0.57)$ & $\mathrm{A}(0.43)$ & $\mathrm{G}(0.57)$ & $\mathrm{A}(0.43)$ \\
\hline 90,636 & $\mathrm{C}(0.70)$ & $\mathrm{A}(0.30)$ & $\mathrm{A}(0.70)$ & $\mathrm{C}(0.30)$ \\
\hline 95,918 & $\mathrm{~A}(0.43)$ & $\mathrm{C}(0.57)$ & $\mathrm{C}(0.59)$ & $\mathrm{A}(0.41)$ \\
\hline 99,784 & $\mathrm{~A}(0.40)$ & $\mathrm{G}(0.60)$ & $\mathrm{G}(0.62)$ & A $(0.38)$ \\
\hline 100,362 & $\mathrm{C}(0.42)$ & $\mathrm{A}(0.58)$ & $\mathrm{A}(0.59)$ & $\mathrm{C}(0.41)$ \\
\hline 100,538 & $\mathrm{G}(0.42)$ & $\mathrm{A}(0.58)$ & $\mathrm{A}(0.61)$ & $\mathrm{G}(0.39)$ \\
\hline
\end{tabular}

are present in the reference, but absent in other individuals, or detecting smaller variations, like SNPs. This method does not identify regions that are absent from the single reference, while these regions might be valuable for clustering the non-reference individuals. Pairwise comparison is able to identify unique regions for both individuals; however, it is difficult to scale to a larger sample size, because every individual has to be compared to every other individual, then the results have to be brought to the same scale.

To take full advantage of next generation sequencing data, a paradigm shift is needed: from focusing on a single reference genome to using a pan-genome, that is, a representation of all genomic content in a certain population, species or phylogenetic clade (Computational Pan-Genomics Consortium, 2018). In this study, we used an ad hoc pan-genomic analysis of the mitochondrial genomes of Fusarium graminearum. The reason for using an ad hoc approach is that pan-genomics is still a young field of research, and as such, there are no clear standards developed yet for analysis, for files or for data sharing. The goal of the analysis was to understand the nature and the dynamics of mitogenome variability, then to identify the implications of these results for mitogenome based population studies or track \& trace implementations. The results of this study can be utilized for the development of suitable data structures and file formats for capturing the variability of mitochondrial pan-genomes.

In this study, we improved the mitochondrial genome reference for F. graminearum strain $\mathrm{PH}-1$, which is recognized as the reference strain of this species for genomic studies 
(Al-Reedy et al., 2012; King et al., 2015; Cuomo et al., 2007). The first mitochondrial genome sequence was produced using Sanger sequencing and primer walking by Al-Reedy et al. (2012). The assembly was improved by King et al. (2015) using NGS reads. This assembly corrected 15 SNPs and 30 indels in the sequence. Here, we present a new assembly, which corrected two more SNPs, complete with a detailed annotation. The introns that were predicted during the annotation process were all verified by RNA-seq data.

The mitochondrial genomes of F. graminearum and F. gerlachii contained the same genes and ORFs in the same orientation. The coding sequences showed high levels of conservations, and all SNPs found in protein coding genes were synonymous substitutions. The genetic variation in the mitochondrial genome could be classified into two groups: small sequence variations (SNPs and short indels) and intron gain and loss. Although, variations resulting from SNPs and short indels were twice as frequent in intergenic regions as in intron regions, about half of the variable sites were located in intron regions. The second type of variation, the presence/absence of introns, accounted for $99.98 \%$ of the length variation between the mitochondrial genomes. In conclusion, the majority of the sequence variation within the species was related to intron regions: either SNPs and short indels or the presence/absence of complete introns. Thus, in mitogenome comparative analysis or pan-genomic studies, special attention should be given to accurately capturing the intron variation, since it is the most informative fraction of the mitogenome.

The annotation of strain CBS 119173 revealed a putative nested intron in cox1-i906. All other strains contain a haplotype that is $1,006 \mathrm{bp}$ long, while this strain contains a haplotype that is 2,084 bp long. The sequence comparison indicates that the additional $1,078 \mathrm{bp}$ region is an intron that was integrated inside the homing endonuclease of the acceptor intron. This putative intron contains an additional HEG, but the annotation pipeline did not identify the sequence as an intron. This type of construct is referred to as a twintron (Copertino \& Hallick, 1991), and it shows that introns and intron encoded genes themselves are susceptible for intron invasions. The question is whether the invading intron has to retain its self-splicing function or the "host" (or acceptor) intron can splice the complete nested construct with its own self-splicing activity.

Most of the introns in F. graminearum are group I introns, and contain homing endonuclease genes (HEGs). Group I introns harboring a functional HEG can spread in a population through homing. Homing is facilitated by the homing endonuclease that cleaves the target gene at a $15-45$ bp recognition site. The resulting double strand break stimulates homologous recombination based DNA repair. Since all copies of the mitochondrial genome that contain the recognition site are susceptible to the homing endonuclease, the only viable template for homologous repair is a genome that contains a copy of the intron. The insertion of the intron into the recognition site modifies the sequence, and it will no longer be recognized by the homing endonuclease.

The mitochondrial genome of F. graminearum shows evidence of heterologous recombination. We recently showed that mitochondrial recombination does also happen in the F. oxysporum species complex (Brankovics et al., 2017). Recombination of the mitochondrial genome in Fusarium appears to be a common phenomenon, since both F. oxysporum and F. graminearum show signs of mitochondrial recombination, despite 
the fact that F. oxysporum is an asexual fungus with a putative parasexual cycle, while F. graminearum is a homothallic species that has an active sexual cycle (Yun et al., 2000). This finding does not prove that mitochondria are not uniparentally inherited in this fungus, but shows that heterologous recombination is widespread enough to be taken into consideration when using mitochondrial sequences for population level studies.

Based on the spreading mechanism of introns, introns could potentially be used for track and trace implementations, where introns could be viewed as "tags". The intron sequences spread through clonal \& sexual reproduction, and through horizontal transfer. Due to the effect of the homing endonuclease, all offspring of a sexual cross would be "tagged" by all the introns that are specific to either parent. The appearance of a new intron in a population or the detection of an intron that is endemic to a region at a new geographic location could be signs of migration or gene flow.

An alternative way to sequencing strains individually is sequencing them in a pool. The pooled sequencing approach is more cost efficient than sequencing the strains separately. The data produced by pooled sequencing of strains from a given population could be viewed as the pan-genomic sequencing reads of that population. In this study, we have demonstrated how sequencing data from pools of strains can be mined for mitochondrial genome variation. Sequencing in pools has already been used to discover rare alleles of nuclear loci (Raineri et al., 2012). This method can be used for finding rare alleles, but it also allows a scalable solution for analyzing complete populations. So far, the application of pooled sequencing data has been used for SNP discovery in nuclear loci from samples (Raineri et al., 2012). However, analyzing mitochondrial genome data of fungi presents some additional challenges. We have demonstrated that besides SNPs, intron presence/absence variation is a major element of the mitogenome variation. To assess what kind of approach can detect intron presence/absence variation and SNP variation, we analyzed the data using a de novo assembly approach followed by a read mapping and SNP-calling approach. The results show that the assembly approach is able to identify sequence differences affecting sequence regions longer than individual sequencing reads, such as, insertions and deletions of intron sequence or long polymorphic sequences, while it is unable to identify SNPs or short indels. Read mapping and SNP calling analysis has to be performed to identify SNPs. This method in turn is unable to identify sequence differences affecting longer sequence regions. For optimal results, a sequential approach is needed for analyzing pooled samples: first, an assembly step to identify introns or larger indels absent from the reference genome, then using both the reference and the newly identified extra regions for read mapping and SNP-calling.

The disadvantages of pooled data are that short indel variation might be missed and linkage between markers is lost when using short read sequencing technologies, although linkage information is not crucial when comparing pan-genomes with each other. Furthermore, pooling large amount of strains could mean the loss of the coverage gap between mitochondrial copies and nuclear copies. This means that nuclear mitochondrial sequences (NUMTs) might affect the results by overestimating the amount of mitochondrial variation (Song et al., 2008). With sufficient caution the effects of NUMTs can be minimized, since they can be identified in the assembly step. In the assembly step, NUMTs 
may appear as separate contigs, as in our example, or as new paths similar to introns with the significant difference that intron segments are joined to the rest of the mitochondrial assembly on both termini, while the flanking nuclear regions of NUMTs would only be joined on one of the termini of the segment. Despite these concerns, the benefit of pooled sequencing of large numbers of strains is that it offers a scalable solution for population or species level comparisons: after establishing a reference sequence, each population or species could then be represented by pan-genomes that are generated from pooled sequencing of multiple strains.

\section{CONCLUSIONS}

We have improved the reference mitochondrial genome sequence for F. graminearum. Intraspecific mitochondrial genome length variations are mainly due to intron presence/absence variation, thus using "intronless" length—subtracting the length of the intron regions from the total mitogenome length—could be a valuable information when comparing species. Mitogenomes are also subject to recombination in both F. graminearum and in F. oxysporum, indicating that it is a common phenomenon in Fusarium. We proposed that introns unique to a single population could potentially be used to track the spread of the given population, because introns can spread through vertical inheritance, recombination and horizontal transfer. We also demonstrated how pooled sequencing of strains can be used for the mitogenome. The usage of pooled sequencing offers a scalable solution for population analysis and for species level comparisons. The results of this study represent an important step towards establishing pan-genomics for mitochondrial genomes.

\section{ADDITIONAL INFORMATION AND DECLARATIONS}

\section{Funding}

This work was supported by the Division for Earth and Life Sciences (ALW) with financial aid from the Netherlands Organization for Scientific Research (NWO, http://www.nwo.nl/) under grant number 833.13.006. The contributions of Cees Waalwijk were financially supported from the MycoKey project (Horizon2020, nr. 678781). The funders had no role in study design, data collection and analysis, decision to publish, or preparation of the manuscript.

\section{Grant Disclosures}

The following grant information was disclosed by the authors:

Netherlands Organization for Scientific Research: 833.13.006.

MycoKey project Horizon2020: 678781.

\section{Competing Interests}

The authors declare there are no competing interests.

\section{Author Contributions}

- Balázs Brankovics conceived and designed the experiments, analyzed the data, prepared figures and/or tables, authored or reviewed drafts of the paper, approved the final draft. 
- Tomasz Kulik conceived and designed the experiments, performed the experiments, analyzed the data, contributed reagents/materials/analysis tools, authored or reviewed drafts of the paper, approved the final draft.

- Jakub Sawicki and Katarzyna Bilska performed the experiments, authored or reviewed drafts of the paper, approved the final draft.

- Hao Zhang authored or reviewed drafts of the paper, approved the final draft.

- G Sybren de Hoog authored or reviewed drafts of the paper, approved the final draft.

- Theo A.J. van der Lee conceived and designed the experiments, performed the experiments, analyzed the data, contributed reagents/materials/analysis tools, authored or reviewed drafts of the paper, approved the final draft.

- Cees Waalwijk conceived and designed the experiments, performed the experiments, contributed reagents/materials/analysis tools, authored or reviewed drafts of the paper, approved the final draft.

- Anne D. van Diepeningen conceived and designed the experiments, performed the experiments, contributed reagents/materials/analysis tools, authored or reviewed drafts of the paper, approved the final draft.

\section{DNA Deposition}

The following information was supplied regarding the deposition of DNA sequences:

The mitochondrial genome sequences described here are accessible via GenBank accession numbers KP966550-KP966561, KR011238, NC_025928, MH412632.

Mitochondrial sequence data for the strains sequenced by third party that were assembled and analyzed in this study are available in the Third Party Annotation Section of the DDBJ/ENA/GenBank databases under the accession numbers TPA: BK010538 -BK010547, and can also be found in the Supplemental Information.

\section{Data Availability}

The following information was supplied regarding data availability:

European Nucleotide Archive: PRJEB18592, PRJEB18596.

\section{Supplemental Information}

Supplemental information for this article can be found online at http://dx.doi.org/10.7717/ peerj.5963\#supplemental-information.

\section{REFERENCES}

Al-Reedy RM, Malireddy R, Dillman CB, Kennell JC. 2012. Comparative analysis of Fusarium mitochondrial genomes reveals a highly variable region that encodes an exceptionally large open reading frame. Fungal Genetics and Biology 49(1):2-14 DOI 10.1016/j.fgb.2011.11.008.

Avila-Adame C, Gómez-Alpizar L, Zismann V, Jones KM, Buell CR, Ristaino JB. 2006. Mitochondrial genome sequences and molecular evolution of the Irish potato famine pathogen, Phytophthora infestans. Current Genetics 49(1):39-46 DOI 10.1007/s00294-005-0016-3. 
Bankevich A, Nurk S, Antipov D, Gurevich AA, Dvorkin M, Kulikov AS, Lesin VM, Nikolenko SI, Pham S, Prjibelski AD, Pyshkin AV, Sirotkin AV, Vyahhi N, Tesler G, Alekseyev MA, Pevzner PA. 2012. SPAdes: a new genome assembly algorithm and its applications to single-cell sequencing. Journal of Computational Biology 19(5):455-477 DOI 10.1089/cmb.2012.0021.

Basse CW. 2010. Mitochondrial inheritance in fungi. Current Opinion in Microbiology 13(6):712-719 DOI 10.1016/j.mib.2010.09.003.

Brankovics B, Van Dam P, Rep M, De Hoog GS, Van der Lee TAJ, Waalwijk C, Van Diepeningen AD. 2017. Mitochondrial genomes reveal recombination in the presumed asexual Fusarium oxysporum species complex. BMC Genomics 18(1):735 DOI 10.1186/s12864-017-4116-5.

Brankovics B, Zhang H, Van Diepeningen AD, Van der Lee TAJ, Waalwijk C, De Hoog GS. 2016. GRAbB: Selective assembly of genomic regions, a new niche for genomic research. PLOS Computational Biology 12(6):e1004753 DOI 10.1371/journal.pcbi.1004753.

Bruen TC, Philippe H, Bryant D. 2006. A simple and robust statistical test for detecting the presence of recombination. Genetics 172(4):2665-2681 DOI 10.1534/genetics.105.048975.

Computational Pan-Genomics Consortium. 2018. Computational pan-genomics: status, promises and challenges. Briefings in Bioinformatics 19(1):118-135 DOI 10.1093/bib/bbw089.

Copertino DW, Hallick RB. 1991. Group II twintron: an intron within an intron in a chloroplast cytochrome b-559 gene. The EMBO Journal 10(2):433-442 DOI 10.1002/j.1460-2075.1991.tb07965.x.

Cuomo CA, Güldener U, Xu J-R, Trail F, Turgeon BG, Di Pietro A, Walton JD, Ma L-J, Baker SE, Rep M, Adam G, Antoniw J, Baldwin T, Calvo S, Chang Y-L, Decaprio D, Gale LR, Gnerre S, Goswami RS, Hammond-Kosack K, Harris LJ, Hilburn K, Kennell JC, Kroken S, Magnuson JK, Mannhaupt G, Mauceli E, Mewes HW, Mitterbauer R, Muehlbauer G, Münsterkötter M, Nelson D, O'Donnell K, Ouellet T, Qi W, Quesneville H, Roncero MIG, Seong K-Y, Tetko IV, Urban M, Waalwijk C, Ward TJ, Yao J, Birren BW, Kistler HC, O'Donnell K, Ouellet T, Qi W, Quesneville H, Roncero MIG, Seong K-Y, Tetko IV, Urban M, Waalwijk C, Ward TJ, Yao J, Birren BW, Kistler HC. 2007. The Fusarium graminearum genome reveals a link between localized polymorphism and pathogen specialization. Science 317(5843):1400-1402 DOI 10.1126/science.1143708.

Desjardins AE. 2006. Fusarium Mycotoxins: chemistry, genetics, and biology. St. Paul: The American Phytopathological Society, 260.

Edgar RC. 2004a. MUSCLE: a multiple sequence alignment method with reduced time and space complexity. BMC Bioinformatics 19(5):113.

Edgar RC. 2004b. MUSCLE: multiple sequence alignment with high accuracy and high throughput. Nucleic Acids Research 32(5):1792-1797 DOI 10.1093/nar/gkh340. 
Ehrlich GD. 2001. The biofilm and distributed genome paradigms provide a new theoretical structure for understanding chronic bacterial infections. In: Interscience conference on antimicrobials agents and chemotherapy (ICAAC). Chicago, IL.

Ehrlich GD, Hu FZ, Post JC. 2004. Role for biofilms in infectious disease. In: Ghannoum M, O’Toole GA, eds. Microbial biofilms. Washington, D.C.: ASM Press, 332-358 DOI 10.1128/9781555817718.ch181-55581-294-5.

Fourie G, Van der Merwe NA, Wingfield BD, Bogale M, Tudzynski B, Wingfield MJ, Steenkamp ET. 2013. Evidence for inter-specific recombination among the mitochondrial genomes of Fusarium species in the Gibberella fujikuroi complex. BMC Genomics 14(1):605 DOI 10.1186/1471-2164-14-605.

Gale LR, Ward TJ, Balmas V, Kistler HC. 2007. Population subdivision of Fusarium graminearum sensu stricto in the Upper Midwestern United States. Phytopathology 97(11):1434-1439 DOI 10.1094/PHYTO-97-11-1434.

Gardiner DM, Stiller J, Kazan K. 2014. Genome sequence of Fusarium graminearum isolate CS3005. Genome Announcements 2(2):e00227-14 DOI 10.1128/JB.00990-12.

Gilmore SR, Gräfenhan T, Louis-Seize G, Seifert KA. 2009. Multiple copies of cytochrome oxidase 1 in species of the fungal genus Fusarium. Molecular Ecology Resources 9(Suppl. 1):90-98 DOI 10.1111/j.1755-0998.2009.02636.x.

Goddard MR, Burt A. 1999. Recurrent invasion and extinction of a selfish gene. Proceedings of the National Academy of Sciences of the United States of America 96(24):13880-13885 DOI 10.1073/pnas.96.24.13880.

Harrison RG. 1989. Animal mitochondrial DNA as a genetic marker in population and evolutionary biology. Trends in Ecology and Evolution 4(1):6-11 DOI 10.1016/0169-5347(89)90006-2.

Haugen P, Simon DM, Bhattacharya D. 2005. The natural history of group I introns. Trends in Genetics 21(2):111-119 DOI 10.1016/j.tig.2004.12.007.

Hebert PDN, Cywinska A, Ball SL, DeWaard JR. 2003. Biological identifications through DNA barcodes. Proceedings of the Royal Society B: Biological Sciences 270(1512):313-321 DOI 10.1098/rspb.2002.2218.

Kim D, Langmead B, Salzberg SL. 2015. HISAT: a fast spliced aligner with low memory requirements. Nature Methods 12(4):357-360 DOI 10.1038/nmeth.3317.

King R, Urban M, Hammond-Kosack MCU, Hassani-Pak K, Hammond-Kosack KE. 2015. The completed genome sequence of the pathogenic ascomycete fungus Fusarium graminearum. BMC Genomics 16:544 DOI 10.1186/s12864-015-1756-1.

Kulik T, Brankovics B, Sawicki J, Van Diepeningen AD. 2016. The complete mitogenome of Fusarium gerlachii. Mitochondrial DNA. Part A, DNA Mapping, Sequencing, and Analysis 27(3):1895-1896 DOI 10.3109/19401736.2014.971275.

Laurent B, Moinard M, Spataro C, Ponts N, Barreau C, Foulongne-Oriol M. 2017. Landscape of genomic diversity and host adaptation in Fusarium graminearum. BMC Genomics 18(203):1-19 DOI 10.1186/s12864-017-3524-x.

Li H, Durbin R. 2009. Fast and accurate short read alignment with Burrows-Wheeler transform. Bioinformatics 25(14):1754-1760 DOI 10.1093/bioinformatics/btp324. 
Li H, Handsaker B, Wysoker A, Fennell T, Ruan J, Homer N, Marth G, Abecasis G, Durbin R. 2009. The sequence alignment/map format and SAMtools. Bioinformatics 25(16):2078-2079 DOI 10.1093/bioinformatics/btp352.

Liu Y, Steenkamp ET, Brinkmann H, Forget L, Philippe H, Lang BF. 2009. Phylogenomic analyses predict sistergroup relationship of nucleariids and Fungi and paraphyly of zygomycetes with significant support. BMC Evolutionary Biology 9:272 DOI 10.1186/1471-2148-9-272.

Marchler-Bauer A, Bryant SH. 2004. CD-Search: protein domain annotations on the fly. Nucleic Acids Research 32(Web Server issue):327-331 DOI 10.1093/nar/gkh454.

Mitchell A, Chang H-Y, Daugherty L, Fraser M, Hunter S, Lopez R, McAnulla C, McMenamin C, Nuka G, Pesseat S, Sangrador-Vegas A, Scheremetjew M, Rato C, Yong S-Y, Bateman A, Punta M, Attwood TK, Sigrist CJA, Redaschi N, Rivoire C, Xenarios I, Kahn D, Guyot D, Bork P, Letunic I, Gough J, Oates M, Haft D, Huang H, Natale DA, Wu CH, Orengo C, Sillitoe I, Mi H, Thomas PD, Finn RD. 2015. The InterPro protein families database: the classification resource after 15 years. Nucleic Acids Research 43(Database issue):D213-D221 DOI 10.1093/nar/gku1243.

Nurk S, Bankevich A, Antipov D, Gurevich AA, Korobeynikov A, Lapidus A, Prjibelski AD, Pyshkin A, Sirotkin A, Sirotkin Y, Stepanauskas R, Clingenpeel SR, Woyke T, McLean JS, Lasken R, Tesler G, Alekseyev MA, Pevzner PA. 2013. Assembling single-cell genomes and mini-metagenomes from chimeric MDA products. Journal of Computational Biology 20(10):714-737 DOI 10.1089/cmb.2013.0084.

Pavesi A, Conterio F, Bolchi A, Dieci G, Ottonello S. 1994. Identification of new eukaryotic tRNA genes in genomic DNA databases by a multistep weight matrix analysis of transcriptional control regions. Nucleic Acids Research 22(7):1247-1256 DOI 10.1093/nar/22.7.1247.

Raineri E, Ferretti L, Esteve-Codina A, Nevado B, Heath S, Pérez-Enciso M. 2012. SNP calling by sequencing pooled samples. BMC Bioinformatics 13:239 DOI 10.1186/1471-2105-13-239.

Smith AM, Heisler LE, St.Onge RP, Farias-Hesson E, Wallace IM, Bodeau J, Harris AN, Perry KM, Giaever G, Pourmand N, Nislow C. 2010. Highly-multiplexed barcode sequencing: an efficient method for parallel analysis of pooled samples. Nucleic Acids Research 38(13):e142 DOI 10.1093/nar/gkq368.

Song H, Buhay JE, Whiting MF, Crandall KA. 2008. Many species in one: DNA barcoding overestimates the number of species when nuclear mitochondrial pseudogenes are coamplified. Proceedings of the National Academy of Sciences of the United States of America 105(36):13486-13491 DOI 10.1073/pnas.0803076105.

Taylor JW. 1986. Fungal evolutionary biology and mitochondrial DNA. Experimental Mycology 10(4):259-269 DOI 10.1016/0147-5975(86)90011-3.

Tettelin H, Masignani V, Cieslewicz MJ, Donati C, Medini D, Ward NL, Angiuoli SV, Crabtree J, Jones AL, Durkin AS, DeBoy RT, Davidsen TM, Mora M, Scarselli M, Margarit y Ros I, Peterson JD, Hauser CR, Sundaram JP, Nelson WC, Madupu R, Brinkac LM, Dodson RJ, Rosovitz MJ, Sullivan SA, Daugherty SC, Haft DH, Selengut J, Gwinn ML, Zhou L, Zafar N, Khouri H, Radune D, Dimitrov G, Watkins 
K, O'Connor KJB, Smith S, Utterback TR, White O, Rubens CE, Grandi G, Madoff LC, Kasper DL, Telford JL, Wessels MR, Rappuoli R, Fraser CM. 2005. Genome analysis of multiple pathogenic isolates of Streptococcus agalactiae: implications for the microbial "pan-genome". Proceedings of the National Academy of Sciences of the United States of America 102(39):13950-13955 DOI 10.1073/pnas.0506758102.

Wang Q, Jiang C, Wang C, Chen C, Xu J-R, Liu H. 2017. Characterization of the two-speed subgenomes of Fusarium graminearum reveals the fast-speed subgenome specialized for adaption and infection. Frontiers in Plant Science 8:140 DOI 10.3389/fpls.2017.00140.

Ward TJ, Clear RM, Rooney AP, O'Donnell K, Gaba D, Patrick S, Starkey DE, Gilbert J, Geiser DM, Nowicki TW. 2008. An adaptive evolutionary shift in Fusarium head blight pathogen populations is driving the rapid spread of more toxigenic Fusarium graminearum in North America. Fungal Genetics and Biology 45:473-484 DOI 10.1016/j.fgb.2007.10.003.

Wick RR, Schultz MB, Zobel J, Holt KE. 2015. Bandage: Interactive visualization of de novo genome assemblies. Bioinformatics 31(20):3350-3352

DOI 10.1093/bioinformatics/btv383.

Yun SH, Arie T, Kaneko I, Yoder OC, Turgeon BG. 2000. Molecular organization of mating type loci in heterothallic, homothallic, and asexual Gibberella/Fusarium species. Fungal Genetics and Biology 31(1):7-20 DOI 10.1006/fgbi.2000.1226.

Zhang H, van der Lee TAJ, Waalwijk C, Chen W, Xu J, Xu J, Zhang Y, Feng J. 2012. Population analysis of the species complex from wheat in China show a shift to Fusarium graminearum more aggressive isolates. PLOS ONE 7(2):e31722 DOI 10.1371/journal.pone.0031722.

Zhao C, Waalwijk C, De Wit P, Tang D, Van der Lee T. 2014. Relocation of genes generates non-conserved chromosomal segments in Fusarium graminearum that show distinct and co-regulated gene expression patterns. BMC Genomics 15(1): 191 DOI 10.1186/1471-2164-15-191. 